CRITICA, Revista Hispanoamericana de Filavofta

Vol. XXI, No. 62 (ngosto 1989): 55-66

\title{
LA HIPÓTESIS GENERALIZADA DEL CONTINUO (HGC) Y SU RELACIÓN CON EL AXIOMA DE ELECCIÓN (AE) *
}

JOSE ALFREDO AMOR

Facultad de Ciencias, UNAM

La llamada Hipótesis del Continuo (HC) es la afirmación: " $2^{\aleph_{0}}=\aleph_{1}$ ", o bien: "el cardinal del continuo es $\aleph_{1}$, el cardinal sucesor de $\aleph_{0}$ ". Otras versiones equivalentes de la Hipótesis del Continuo son las siguientes:

i) No hay cardinal entre el cardinal del conjunto $\mathbb{N}$ de los números naturales (que es $\aleph_{0}$ ) y el cardinal del continuo o del conjunto $\mathbb{R}$ de los números reales (que es $2^{\aleph_{0}}$ ).

ii) Para todo $S \subseteq \mathbb{R}$ ( $S$ es finito o $S \equiv \mathbb{N}$ o $S \equiv \mathbb{R}$ ).

iii) $\neg \exists x(\mathbb{N} \prec x$ y $x \prec \mathbb{R})$.

iv) $|\mathbb{R}|=\aleph_{1}$;

donde el símbolo $\equiv$ denota una biyección entre los conjuntos. Si hay una inyección de $x$ en $z$ lo denotaremos $x \preceq z$ y diremos que $x$ está dominado por $z ; x \prec z$ denota la existencia de una inyección de $x$ en $z$, pero que no hay biyección entre $x$ y $z$, en tal caso diremos que $x$ está estrictamente dominado por $z$. $|\mathbb{R}|$ denota el cardinal del conjunto $\mathbb{R}$ de los números reales. El símbolo $\neg$ denota negación.

- Una versión previa de este trabajo fue presentada en un seminario sobre teoría de conjuntos con los profesores Carlos Álvarez y Raúl Orayen, a quienes agradezco sus observaciones; especialmente la revisión detallada del trabajo por el profewor Orayen y sus sugerencias. 
Georg Cantor conjeturó la verdad de esta hipótesis y su demostrabilidad en la teoría de conjuntos, así como la de su generalización, la llamada Hipótesis Generalizada del Continuo (HGC): "Si $K$ es un cardinal transfinito y $2^{K}$ denota el cardinal del conjunto potencia de un conjunto de cardinal $K$, y $K^{+}$denota el cardinal sucesor de $K$, entonces $2^{K}=K^{+"}$. Las correspondientes versiones equivalentes de la HGC son las siguientes, donde $A$ es un conjunto infinito:

i') No hay cardinal entre el cardinal del conjunto $A$ y el cardinal del conjunto potencia de $A, P(A)$, también llamado conjunto de partes de $A$ o de subconjuntos de $A$.

ii') Para todo $S \subseteq P(A)(S \prec A$ o $S \equiv A$ o $S \equiv P(A))$.

iii') $\forall A \neg \exists x(A \prec x$ y $x \prec P(A))$.

iv') $|P(A)|=|A|^{+}$.

Es claro que: $\mathrm{HGC} \Rightarrow \mathrm{HC}$.

Gödel probó en 1939 la consistencia relativa de la HGC (y por lo tanto la consistencia relativa de la $\mathrm{HC}$ ) con la teoría de conjuntos $\mathrm{ZF}+\mathrm{AE}$ (Zermelo-Fraenkel con Axioma de Elección); lo que Gödel probó es que: "Si ZF + AE tiene un modelo ( $y$ tener un modelo es equivalente a ser consistente), entonces hay un modelo de ZF + AE + HGC" [1]. Cohen probó, en 1963 (mediante la técnica de forcing), la consistencia relativa de la negación de la HC (y por lo tanto la consitencia relativa de la negación de la $\mathrm{HGC}$ ) con la teoría $\mathrm{ZF}+\mathrm{AE}$, es decir, que: "Si $\mathrm{ZF}+\mathrm{AE}$ tiene un modelo, entonces hay un modelo de $\mathrm{ZF}+\mathrm{AE}+\neg \mathrm{HC}$ " [2]. De lo anterior, si la teoría de conjuntos $\mathrm{ZF}+\mathrm{AE}$ es consistente, entonces no prueba ni refuta la HGC, es decir, la HGC es indecidible en ZF + AE.

Por otro lado, Gödel probó, también en 1939, la consistencia relativa del Axioma de Elección (AE) con la teoría de conjuntos ZF (Zermelo-Fraenkel sin Axioma de Elección), esto es: "Si ZF tiene un modelo entonces hay un modelo de $\mathrm{ZF}+\mathrm{AE}$ ". Cohen probó, también en 1963, la consistencia relativa de la negación 
del AE con ZF o sea que: "Si ZF tiene algún modelo, entonces hay un modelo de $\mathrm{ZF}+\neg \mathrm{AE}$." Así pues, si la teoría de los conjuntos $\mathrm{ZF}$ es consistente, entonces no prueba ni refuta el $\mathrm{AE}$, es decir, el $\mathrm{AE}$ es indecidible en $\mathrm{ZF}$.

Combinando los resultados de Gödel y Cohen de los párrafos anteriores, es fácil ver que: si ZF tiene un modelo, entonces tanto ZF + HGC como ZF + $\neg$ HGC tienen un modelo; luego, si la teoría ZF es consistente, entonces en ZF no es posible probar ni refutar la HGC; es decir, ésta es indecidible en ZF.

La HGC y el AE son las más famosas afirmaciones indecidibles en ZF; pero, ¿cuál es la relación entre ellas? Es claro de lo anterior que si ZF es consistente, hay modelos de ZF donde sean verdaderos tanto $\mathrm{AE}$ como $\neg \mathrm{HC}$. De esto se desprende que si $\mathrm{ZF}$ es consistente, entonces $\mathrm{ZF} \not / \mathrm{AE} \Rightarrow \mathrm{HC}$ y, por lo tanto, que ZF $\not$ AE $\Rightarrow$ HGC. Es decir, el AE no implica (en ZF) la HC ni la HGC.

Ahora la cuestión es: ¿HGC implica AE, en la teoría ZF? La respuesta es isí! Si se da una definición adecuada de cardinal de un conjunto, la cual no dependa del AE como la definición usual, y una formulación adecuada de HGC equivalente a la tradicional, es muy fácil demostrar en $\mathrm{ZF}$ que: $\mathrm{HGC} \Rightarrow \mathrm{AE}$. Con este resultado, al cual representamos como:

$$
\mathrm{ZF} \vdash \mathrm{HGC} \Rightarrow \mathrm{AE},
$$

se tiene claramente que:

$$
\text { no hay modelo de } \mathrm{ZF}+\mathrm{HGC}+\neg \mathrm{AE} \text {. }
$$

Antes de demostrar el resultado mencionado lo usaremos para mostrar que, suponiendo la consistencia de ZF, también hay modelos de $\mathrm{ZF}+\neg \mathrm{AE}+\neg \mathrm{HGC}$, ya que por el resultado de Cohen: " $\mathrm{ZF}$ tiene un modelo $\Rightarrow \mathrm{ZF}+\neg \mathrm{AE}$ tiene un modelo" y como $\mathrm{ZF} \vdash \neg \mathrm{AE} \Rightarrow \neg \mathrm{HGC}$, se tiene que: " $\mathrm{ZF}+\neg \mathrm{AE}$ tiene un modelo $\Rightarrow \mathrm{ZF}+\neg \mathrm{AE}+\neg \mathrm{HGC}$ tiene un modelo". De estos dos resultados tenemos que: "ZF tiene un modelo" $\Rightarrow$ $\mathrm{ZF}+\neg \mathrm{AE}+\neg \mathrm{HGC}$ tiene un modelo". 
A continuación definiremos cardinal de un conjunto de modo tal que la definición no dependa del $\mathrm{AE}$, como la definición usual, si bien sí dependerá del Axioma de Regularidad o de buena fundación, el cual está justificado en la base intuitiva de la teoría ZF, que considera a los conjuntos construidos a partir de sus elementos que a su vez son conjuntos. La complejidad de este proceso constructivo está indexada con los números ordinales: cualquier conjunto tendrá una determinada complejidad constructiva mayor que las de sus elementos, y esta complejidad constructiva está asociada con un único número ordinal.

Usaremos el concepto de rango de un conjunto, el cual es el número ordinal que intuitivamente representa su complejidad constructiva, así como el Axioma de Regularidad (AR), que podemos enunciar como: "Todo conjunto tiene un rango".

Definición. Si $A$ es un conjunto, definimos el cardinal de $A$, denotado $|A|$ como:

$$
|A|=\left\{\begin{array}{l}
\text { El menor ordinal biyectable con } A, \\
\text { si } A \text { es bien ordenable. } \\
\text { El conjunto de todos los conjuntos } \\
\text { biyectables con } A \text { y de rango míni- } \\
\text { mo; en otro caso. }
\end{array}\right.
$$

Con esta definición de cardinal de un conjunto, si se cumple el AE, o equivalentemente, si todo conjunto es bien ordenable, todo cardinal de un conjunto será un ordinal, como en la definición usual; y si el AE no se cumple, habrá cardinales de conjuntos que sean cardinales ordinales y habrá cardinales de conjuntos que sean cardinales no-ordinales, pues $O \in K$ para todo cardinal ordinal $K>O$ y $O \notin K$, para todo cardinal $K$ del segundo tipo. Con esta definición, las propiedades usuales de cardinales funcionan bien, por ejemplo: $|A|=|B|$ si y sólo si $A \equiv B$, así como la aritmética cardinal; $|A| \leq|B|$ y $|A|<|B|$ se definen como es usual y el $<$ es un orden parcial, 
aunque los cardinales podrían no formar un orden lineal. Véase [3], p. 220.

Hay que mencionar que, sin suponer $\mathrm{AE}$, es necesario suponer AR para poder definir $|A|$ de modo que se cumpla: $|A|=$ $|B|$ si y sólo si $A \equiv B$; ya que $\sin A E$ ni $A R$, no es posible definir cardinal de un conjunto de modo que se cumpla lo anterior. Véase [5] p. 98.

Para definir el cardinal sucesor de un cardinal, usaré el conocido Teorema de Hartog ([3] p. 195 o [4] p. 30), el cual es independiente del AE.

Teorema de Hartog. Para todo conjunto $A$ hay un mínimo cardinal ordinal no dominado por $A$ y sí dominado por $P(P(A \times A))$.

Prueba:

Sea $\lambda=\{\alpha: \alpha$ es ordinal y $\alpha \preceq A\}$.

Si $\lambda$ es un conjunto, es inmediato verificar que es cardinal ordinal, que $\lambda \npreceq A$ y que es el mínimo con tales propiedades. Para verificar su existencia, consideremos:

$$
W=\{r \subseteq A \times A: \exists z \subseteq A(r \text { bien ondena } z)\} .
$$

Entonces $W \subseteq P(A \times A)$. Defino $H: W \rightarrow O R$, donde $O R$ denota la clase de los ordinales y $H(r)$ sea el único ordinal $\alpha$ tal que $\langle\operatorname{dom}(r), r\rangle \simeq\langle\alpha, \in\rangle$, justificado por el Teorema de Enumeración: para todo buen orden existe un único ordinal con el cual es isomorfo. Es inmediato verificar que $h[W]=\lambda$ de donde ya que $W$ es conjunto y $H$ es función, $\lambda$ es conjunto por el Axioma de Reemplazo. Ahora definamos $F: \lambda \rightarrow P(P(A \times A))$ tal que si $\alpha \in \lambda, F(\alpha)=H^{-1}(\alpha)=$ $\{r \in W: H(r)=\alpha\}$; es claro que $F$ es inyectiva, de donde $\lambda \preceq P(P(A \times A))$.

Definición. Para todo cardinal $K, K^{+}$denota el menor cardinal ordinal no dominado por $K$, dado por el Teorema de Hartog para $A=K$ (también conocido como el número de Hartog de $K$ ). 
Corolario. a) Si $K$ es cardinal ordinal, $K^{+}$es el menor de los cardinales ordinales mayores que $K$, es decir, su sucesor.

b) Para todo cardinal $K, K^{+} \leq|P(P(K \times K))|=2^{2^{K^{2}}}$.

Ahora bien, la HGC* la formularemos así:

$H G C^{*}$ : Para todo $K$, cardinal ordinal transfinito, $2^{K}=K^{+}$, o bien para todo ordinal $\alpha, 2^{\aleph_{\alpha}}=\aleph_{\alpha+1}$.

Esta formulación podrá parecer una restricción a la formulación tradicional, pues se refiere a los cardinales ordinales transfinitos, en vez de referirse a todos los cardinales transfinitos; además con las definiciones dadas, si $K$ es un cardinal no-ordinal, $K^{+}$es un cardinal ordinal, pero pudiera no ser comparable con $K$. Así pues, nuestra formulación y la tradicional son muy diferentes. Sin embargo, son equivalentes, lo cual mostraremos enseguida. Podemos establecer como la formulación tradicional, a la que denotaremos HGC, la versión iii') dada al principio de este artículo:

$H G C: \forall A \neg \exists x(A \prec x$ y $x \prec P(A))$,

o bien $\quad \forall K \neg \exists \lambda\left(K<\lambda\right.$ y $\left.\lambda<2^{K}\right)$,

en términos de cardinales, con la relación menor dada por la dominancia estricta.

El resultado principal de este trabajo mostrará que nuestra formulación $\mathrm{HGC}^{*}$ implica, en $\mathrm{ZF}$, al $\mathrm{AE}$ y con $\mathrm{AE}$, las dos formulaciones son claramente equivalentes, por lo cual nuestra formulación $\mathrm{HGC}^{*}$ implica, en ZF, a la formulación tradicional HGC. Para mostrar la implicación inversa, daremos antes un sencillo lema:

Lema 1 . Si $K$ es un cardinal cualquiera, $\lambda$ un cardinal ordinal y $K \cdot \lambda \leq K+\lambda$, entonces $K$ y $\lambda$ son comparables.

Prueba: Sean $A, B$ tales que $K=|A|, \lambda=|B|, A \cap B=\phi$ y supongamos que $K \cdot \lambda \leq K+\lambda$. Entonces, de acuerdo con la 
definición usual de producto y de suma de cardinales, $A \times B \preceq$ $A \cup B$ y sea $f$ una inyección correspondiente. Si hay un $z \in \vec{A}$ tal que $f[\{z\} \times B] \subseteq A$, entonces $B \equiv\{z\} \times B \preceq A$, de donde $\lambda \leq K$.

Si no hay un $z \in A$ tal que $f[\{z\} \times B] \subseteq A$, entonces $\forall z \in A \exists w \in B$ tal que $f(\langle z, w\rangle) \notin A$ y entonces $f(\langle z, w\rangle) \in$ $B$; sea $g: A \rightarrow B$ tal que $g(z)$ es el menor $w \in b$ tal que $f(\langle z, w\rangle) \in B$, con un buen onden dado para $B$, el cual existe porque $\lambda=|B|$ es cardinal ordinal. Sea $h: A \rightarrow B$ tal que $h(z)=f(\langle z, g(z)\rangle)$. Claramente, $h$ es inyectiva, ya que $f$ lo es y, por tanto, $A \preceq B$, de donde $K \leq \lambda$.

Así pues, $K \leq \bar{\lambda}$ o $\lambda \leq K$ y, así, $K$ y $\lambda$ son comparables.

Proposición 1. ZF $\vdash$ HGC $\Rightarrow$ HGC*.

Supongamos HGC y sea $K$ un cardinal ordinal transfinito. Como $2^{K}<2^{2^{K}}$ por el teorema de Cantor, y $K^{+} \leq 2^{2^{K}}$ por el corolario del Teorema de Hartog, ya que $K^{2}=K$ para todo cardinal ordinal transfinito, entonces:

$$
\begin{aligned}
2^{K} \leq 2^{K}+K^{+} \leq 2^{K} \cdot K^{+} \leq 2^{2^{K}} \cdot 2^{2^{K}} & =2^{\left(2^{K} \cdot 2\right)} \\
& =2^{2^{K+1}} \\
& =2^{2^{K}}
\end{aligned}
$$

de donde

$$
2^{K} \leq 2^{K}+K^{+} \leq 2^{K} \cdot K^{+} \leq 2^{2^{K}} .
$$

Analizare la segunda desigualdad (1):

Si en (1) se cumple la igualdad, entonces se cumple el "mayor o igual", entonces, por el Lema $1,2^{K}$ y $K^{+}$son comparables:

Si $2^{K} \leq K^{+}$, entonces $2^{K}$ será cardinal ordinal y $K<$ $2^{K} \leq K^{+}$, de donde $2^{K}=K^{+}$, pues entre $K$ y $K^{+}$, no hay otro cardinal ordinal. Si $K^{+} \leq 2^{K}$, entonces $K<K^{+} \leq 2^{K}$, de donde, por HCC, $K^{+}=2^{K}$. 
Si en (1) se cumple el "menor estricto", tengo $2^{K} \leq 2^{K}+$ $K^{+}<2^{2^{K}}$, de donde, por la HGC, $2^{K}=2^{K}+K^{+}$, pero entonces, $K<K^{+} \leq 2^{K}$ y, por la HGC, $K^{+}=2^{K}$.

Así, en cualquier caso, $2^{K}=K^{+}$.

Habiendo probado que la formulación tradicional HGC implica nuestra formulación $\mathrm{HGC}^{*}$, veamos el resultado final de este trabajo: $\mathrm{HGC}^{*} \Rightarrow \mathrm{AE}$, con lo cual tendremos $\mathrm{HGC} \Rightarrow \mathrm{AE}$ y además la equivalencia entre HGC y HGC*.

Lema 2. El conjunto potencia de un conjunto bien ordenable es bien ordenable si y sólo si $\mathrm{AE}$.

Este lema es una de las muchas equivalencias del $\mathrm{AE}$ (en ZF) y su demostración es un tanto técnica, por lo que la incluyo como apéndice de este trabajo.

Proposicion 2. ZF $\vdash \mathrm{HGC}^{*} \Rightarrow \mathrm{AE}$.

Probaremos (en ZF) que HGC* implica que el conjunto potencia de un conjunto bien ordenable es bien ordenable. Esto es suficiente por el lema anterior. Supongamos HGC*: $\forall K$ cardinal ordinal, $2^{K}=K^{+}$. Sea $A$ un conjunto bien ordenable. Entonces $|A|=K$ para algún cardinal ordinal $K$ y $A \equiv K$. Sabemos entonces que $P(A) \equiv P(K)$, de donde $|P(A)|=|P(K)|=2^{K}=K^{+}$. Así pues, $|P(A)|=K^{+}$, por lo que $P(A) \equiv K^{+}$, pero $K^{+}$es un cardinal ordinal, por lo cual $P(A)$ es bien ordenable con el buen orden inducido por medio de la biyección, del buen orden de $K^{+}$.

Corolario. ZF $\vdash$ HGC $\Rightarrow$ AE y ZF $\vdash$ HGC $\Leftrightarrow$ HGC*.

A la luz de esta demostración, vemos que lo que afirma la HGC es que: "el cardinal del conjunto potencia de un conjunto bien ordenable $A$ (que es $2^{K}$ si $|A|=K$ ), es un ordinal", pero iesto es equivalente al AE! La HGC afirma todavía algo más, no sólo que tal cardinal es un ordinal, sino que es $K^{+}$, el cardinal ordinal sucesor del cardinal del conjunto bien ordenado $A$. 


\section{APÉNDICE}

Lema 2 (Rubin, 1960). El conjunto potencia de un conjunto bien ordenable es bien ordenable si y sólo si AE.

$\Leftarrow$ ) Por AE, todo conjunto es bien ordenable (Teorema del Buen Orden).

$\Rightarrow)$ Probaremos que todo conjunto es bien ondenable. Por el Axioma de Regularidad (uno de los axiomas de ZF), $\forall x \exists \alpha(x \subseteq$ $V_{\alpha}$ ), donde $\alpha$ es ordinal y $V_{\alpha}$ está definido por recursión como: $V_{0}=\phi, V_{\alpha+1}=P\left(V_{\alpha}\right)$ y, si $\gamma$ es ordinal límite, $V_{\gamma}=\underset{\beta<\gamma}{\bigcup} V_{\beta}$. Definimos el rango de $x$ como el menor ondinal $\alpha$ tal que $x \subseteq$ $V_{\alpha}$. Entonces será suficiente probar que todo $V_{\alpha}$ es bien ordenable, por inducción transfinita sobre $\alpha$ ordinal: $V_{0}=\phi$, es obvio.

Supóngase $V_{\alpha}$ bien ordenable. Entonces $V_{\alpha+1}=P\left(V_{\alpha}\right)$ es bien ordenable por la hipótesis inductiva y la hipótesis del Lema.

Sea $\gamma$ límite, $V_{\gamma}=U\left\{V_{\beta}: \beta<\gamma\right\}$ y supóngase que cada $V_{\beta}$ es bien ordenable, para $\beta<\gamma$. iNo se puede asegurar en general que la unión de bien ordenables sea bien ordenable!, a menos que definamos los buenos óndenes de $V_{\beta}$, para $\beta<\gamma$, de un modo uniforme y acumulativo.

Definiremos una función:

$$
g: \gamma \rightarrow\left\{r: r \text { bien ordena a } V_{\beta}, \beta<\gamma\right\},
$$

tal que $\forall \beta<\gamma,\left\langle V_{\beta}, g(\beta)\right\rangle$ es un buen orden. Con esta $g$ podemos definir, si $\rho(u)$ denota al rango de $u$, el siguiente orden:

$$
\begin{array}{r}
\left\{\langle u, v\rangle: u, v \in V_{\gamma} \text { y }[\rho(u)<\rho(v) 6(\rho(u)=\rho(v)\right. \\
\mathrm{y}\langle u, v\rangle \in g(\rho(u)+1))]\}
\end{array}
$$

el cual es claramente un buen onden para $V_{\gamma}$. Nótese que $u \in$ $V_{\rho(u)+1}$.

Sea $K=\left(\underset{\beta<\gamma}{\cup}\left|V_{\beta}\right|\right)^{+}$; este es un cardinal ordinal, pues por hipótesis inductiva $\forall \beta<\gamma V_{\beta}$ es bien ordenable y $\left|V_{\beta}\right|$ es 
cardinal ordinal. Sea $r$ un buen orden para $P(K)$, el cual existe por hipótesis. Definimos ahora la $g$ por recursión sobre $\beta<\gamma$ :

i) $g(0)=0$.

ii) Definido $g(\beta) \operatorname{con} \beta<\gamma$, sea $\xi$ el tipo de orden del buen orden $\left\langle V_{\beta}, g(\beta)\right\rangle$ y sea $f$ el isomorfismo: $\left\langle V_{\beta}, g(\beta)\right\rangle \cong$ $\langle\xi, \epsilon\rangle$. Debe ser claro que $\xi<K$, por definición de $K$. Pongamos entonces $g(\beta+1)=$ el único buen orden de $V_{\beta+1}=P\left(V_{\beta}\right)$, inducido por $f$ y por el buen orden $r$ de $P(K)$; es decir, definamos:

$$
g(\beta+1)=\left\{\langle u, v\rangle: u, v \subseteq V_{\beta} \mathbf{y}\langle f[u], f[v]\rangle \in r\right\}
$$

iii) Definido $g(\delta)$ con $\delta<\eta<\gamma$, con $\eta$ límite, definimos:

$$
\begin{gathered}
g(\eta)=\left\{\langle u, v\rangle: u, v \in V_{\eta} \mathrm{y}[\rho(u)<\rho(v) 6(\rho(v)=\rho(u)\right. \\
\mathrm{y}\langle u, v\rangle \in g(\rho(u)+1))] .\}
\end{gathered}
$$

\section{REFERENCIAS}

1. K. Gödel, La consistencia del Axioma de Elección y de la Hipótesis Generalizada del Continuo con los axiomas de la teorta de conjuntos, Obras completas, Alianza Editorial, 1981.

2. P. J. Cohen, Set Theory and the Continuum Hypothesis. Ed. Benjamin, Nueva York, 1966.

3. H. B. Enderton, Elements of Set Theory, Academic Press, 1979.

4. K. Kunen, Set Theory (An Introduction to Independence Proofs), North Holland, 1980.

5. A. Fraenkel y Y. Bar-Hillel, Foundations of Set Theory, North Holland, 1973. 


\section{SUMMARY}

The so called Generalized Continuum Hypothesis (GCH) is the sentence: "If $A$ is an infinite set whose cardinal number is $K$ and $2^{K}$ denotes the cardinal number of the set $P(A)$ of subsets of $A$ (the power set of $A$ ), and $K^{+}$denotes the succesor cardinal of $K$, then $2^{K}=K^{+n}$.

The Continuum Hypothesis $(\mathrm{CH})$ asserts the particular case $K=\aleph_{0}$. It is clear that $\mathrm{GCH}$ implies $\mathrm{CH}$.

Another equivalent version of $\mathrm{GCH}$, is the sentence: "Any subset of the set of subsets of a given infinite set is or of cardinality less or equal than the cardinality of the given set, or of the cardinality of all the set of subsets".

Gödel in 1939, and Cohen in 1963, settled the relative consistency of the Axiom of Choice (AC) and of its negation not-AC, respectively, with respect to the Zermelo-Fraenkel set theory (ZF). On the other hand, Gödel in 1939, and Cohen in 1963 settled too, the relative consistency of GCH , $\mathrm{CH}$ and of its negations not-GCH, not-CH, respectively, with respect to the Zermelo-Fraenkel set theory with the Axiom of Choice (ZF + AC or ZFC). From these results we know that GCH and AC are undecidable sentences in ZF set theory and indeed, the most famous undecidable sentences in ZF; but, Which is the relation between them?

From the above results, in the theory $\mathrm{ZF}+\mathrm{AC}$ is not demonstrated $\mathrm{GCH}$; it is clear then that AC doesn't imply GCH in ZF theory. But does GCH implies AC in ZF theory? The answer is yes! or equivalently, there is no model of $\mathrm{ZF}+\mathrm{GCH}+$ not-AC.

$A$ very easy proof can be given if we have an adecuate definition of cardinal number of a set, that doesn't depend of AC but depending from the Regularity Axiom, which asserts that all sets have a range, which is an ordinal number asociated with its constructive complexity. We define the cardinal number of $A$, denoted $|A|$, as follows:

$$
|A|=\left\{\begin{array}{l}
\text { The least ordinal number equipotent with } \\
A, \text { if } A \text { is well orderable } \\
\text { The set of all sets equipotent with } A \text { and of } \\
\text { minimum range, in other case. }
\end{array}\right.
$$

It is clear that without AC, may be not ordinal cardinals and all cardinals are ordinal cardinals if all sets are well orderable (AC). Now we formulate:

$$
\mathrm{GCH}^{*} \text { : For all ordinal cardinal } K, 2^{K}=K^{+}
$$

In the paper is demonstrated that this formulation $\mathrm{GCH}^{*}$ is implied by the traditional one, and indeed equivalent to it.

Lemma. The power set of any well orderable set is well orderable if and only if $\mathrm{AC}$. 
This is one of the many equivalents of AC in ZF, due to Rubin, 1960.

Proposition. In $\mathrm{ZF}$ is a theorem: $\mathrm{GCH}^{*}$ implies AC.

Supose $\mathrm{GCH}^{*}$. Let $A$ be a well orderable set; then $|A|=K$ an ordinal cardinal, so $A$ is equipotent with $K$ and then $P(A)$ is equipotent with $P(K)$; therefore $|P(A)|=|P(K)|=2^{K}=K^{+}$. But then $|P(A)|=$ $K^{+}$and $P(A)$ is equipotent with $K^{+}$and $K^{+}$is an ordinal candinal; therefore $P(A)$ is well orderable with the well order induced by meane of the bijection, from the well order of $K^{+}$.

Corolary. In $\mathrm{ZF}$ are theorems: GCH implies $\mathrm{AC}$ and $\mathrm{GCH}$ is equivalent to $\mathrm{GCH}^{*}$.

We see from this proof, that GCH asserts that the cardinal number of the power set of a well orderable set $A$ is an ordinal, which is equivalent to $\mathrm{AC}$, but GCH asserts also that that ordinal cardinal is $|A|^{+}$, the ordinal cardinal succesor of the ordinal cardinal of the well orderable set $A$. 